\title{
Role of the Low Amplitude Potential in the Initial P Wave Signal-Averaged Elestrogram in Sick Sinus Syndrome
}

\author{
Hidehito Takase, MD; Shinobu Imai, MD; Fumio Saito, MD; \\ Hiroshi Yagi, MD; Toshio Kushiro, MD
}

\begin{abstract}
Background The cause of abnormally low amplitude of the initial $\mathrm{P}$ wave signal-averaged electrocardiogram (P-SAECG) in patients with sick sinus syndrome (SSS) is unknown.

Methods and Results Thirteen normal patients (Group C) and 33 with SSS (Group S) were examined. The root mean square amplitude for the initial $30 \mathrm{~ms}$ (EP30) and the duration of below- $4 \mu \mathrm{V}$ signals of the filtered $\mathrm{P}$ waves (ED4) were measured using the P-SAECG. The interval from an atrial potential on the sinus-node electrogram (SNE) to $\mathrm{P}$ wave onset (AS-P), and the interval from the $\mathrm{P}$ wave to the atrial potential on the His-bundleelectrogram $(\mathrm{P}-\mathrm{AH})$ were measured in the electrophysiological study. The sino-atrial conduction time was measured by a conventional method (indirect sino-atrial conduction time (SACTi)) and using SNE (direct sino-atrial conduction time (SACTd)). The EP30 was significantly lower and the ED4 significantly longer in Group S. The AS-P was significantly longer in Group $\mathrm{S}(\mathrm{p}<0.01)$, but the P-AH was not different. In Group S, the AS-P was significantly correlated with EP30 and ED4 ( $<<0.01)$, but the P-AH was uncorrelated. The SACTi was significantly correlated with EP30 and ED4 ( $<<0.05)$, but the SACTd was uncorrelated.

Conclusion The abnormality of the initial portion of the P-SAECG observed in SSS appears to be due to disturbed conduction through the atrial myocardium around the sino-atrial node. (Circ J 2006; 70: 985-990)
\end{abstract}

Key Words: Atrial conduction; P wave signal-averaged electrocardiogram; Sick sinus syndrome; Sino-atrial conduction; Sinus node eloectrogram

$\mathbf{T}$ he $\mathrm{P}$ wave signal-averaged electrocardiogram ( $\mathrm{P}$ SAECG) is an averaged recording of the $\mathrm{P}$ wave on the body surface electrocardiogram (ECG), which enables noninvasive recording of abnormally low-amplitude electrical activities of the atrium in patients with atrial fibrillation (AF). P-SAECG can help to identify patients at risk of paroxysmal $\mathrm{AF}^{1-3}$ and can predict recurrence after cardioversion of persistent $\mathrm{AF}^{4}$ It has recently been reported that the P-SAECG of patients with sick sinus syndrome (SSS) has a characteristic feature: abnormally low amplitude of the initial portion of the filtered $\mathrm{P}$ waves The precise cause of this abnormality has not been determined. The present study aimed to clarify the cause and clinical significance of this abnormality. P-SAECG data were retrospectively compared with the results of an electrophysiological study using a direct sinus-node electrogram (SNE),6.7

\section{Methods}

\section{Subjects}

The subjects were 13 patients with normal sino-atrial node function (Group C; 3 females, 10 males, age 29-64 years [mean: $51 \pm 13$ ]) and 33 patients with SSS (Group S; 15 females, 18 males, age 37-86 years [63 111$]$ ) who underwent P-SAECG and electrophysiological study (EPS) using

(Received February 10, 2006; revised manuscript received May 18, 2006; accepted June 6, 2006)

Division of Cardiovascular Medicine, Department of Medicine, Nihon University School of Medicine, Tokyo, Japan

Mailing address: Shinobu Imai, MD, Division of Cardiovascular Medicine, Department of Medicine, Nihon University School of Medicine, 1-8-13 Kandasurugadai, Chiyoda-ku, Tokyo 101-8309,

Japan. E-mail: imai@med.nihon-u.ac.jp the SNE. Patients in Group C had Wolff-Parkinson-White syndrome (5 cases), atrial flutter (6 cases), atrioventricular nodal reentry tachycardia ( 2 cases) or atrial tachycardia (1 case); none showed bradycardia. Patients assigned to Group S had symptoms of bradycardia and had a history of cardiac arrest $(\geq 3 \mathrm{~s})$ or persistent bradycardia $(<40 / \mathrm{min})$. According to Rubenstein's classification, in Group S there were 6 patients with Rubenstein type I, corresponding to sinus bradycardia, 12 with type II, corresponding to sinoatrial block, and 17 with type III, corresponding to paroxysmal AF. In Group C, 3 patients had underlying hypertension and 2 patients had underlying diabetes mellitus, compared with 10 and 5, respectively, in Group S. All cardioactive drugs had been discontinued for at least 5 halflives before the P-SAECG and EPS.

\section{Investigations}

$P$-SAECG In accordance with the method reported elsewhere, the P-SAECG was recorded from a modified X Y and Z lead system using a VCM-3000 (Fukuda Denshi Co, Ltd, Japan). The standard I lead was used as the $\mathrm{X}$ lead, the $\mathrm{aVF}$ lead as the $\mathrm{Y}$ lead, and the precordial $\mathrm{V}_{1}$ lead as the $\mathrm{Z}$ lead. The signal from each lead was passed through a band-pass filter passing 30-400 Hz. Signals of more than 200 beats were averaged on a trigger point within a filtered $\mathrm{P}$ wave. Two parameters that indicate abnormally low amplitude of the early-phase filtered $\mathrm{P}$ wave on the PSAECG were measured in the vector magnitude lead: (1) root mean square voltages for the initial $30-\mathrm{ms}$ of the filtered P wave (EP30), and (2) the shortest duration from the onset up to $4 \mu \mathrm{V}$ of the filtered $\mathrm{P}$ wave (ED4).

P-SAECG records in which the mean noise level exceeded $0.5 \mu \mathrm{V}$ were excluded from the analysis. The starting 
Group C

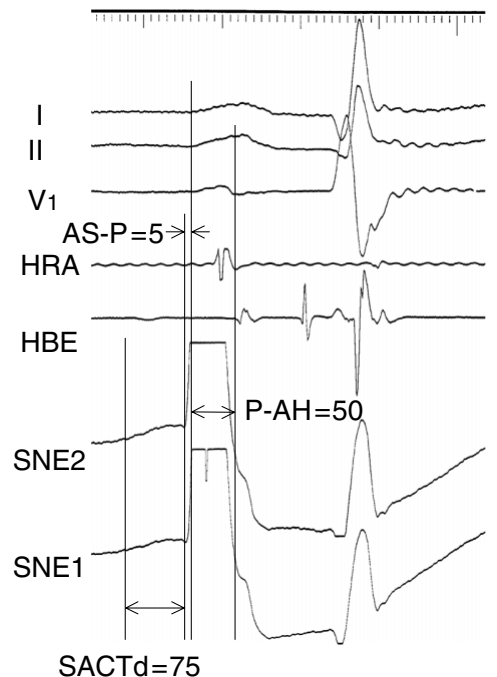

Group S

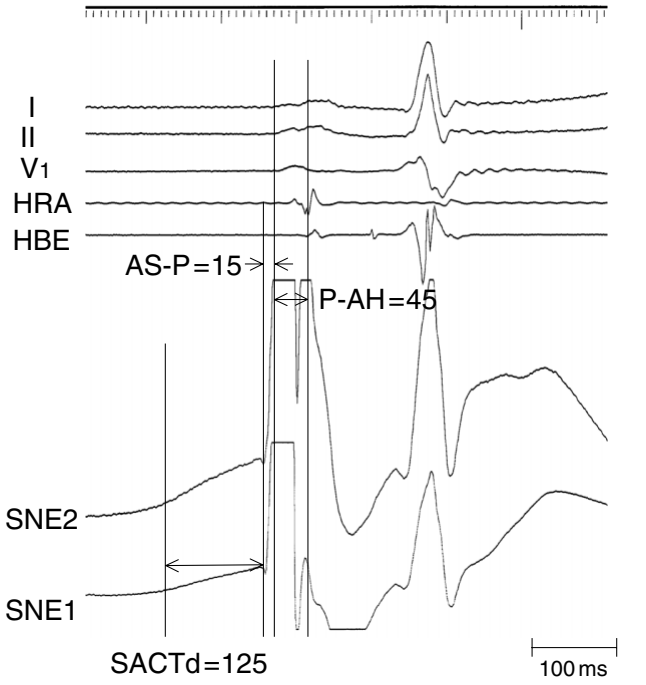

Fig 1. intracardiac electrograms from Group C (Left) and Group S (Right). From top down are shown the body surface ECG leads I, II and $\mathrm{V}_{1}$, HRA recordings, HBE and SNE. Group S has a longer SACTd and AS-P than Group C (see text for abbreviations).

\section{Group C}

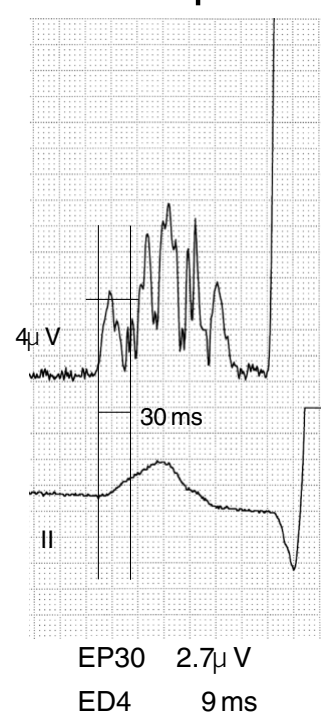

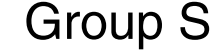

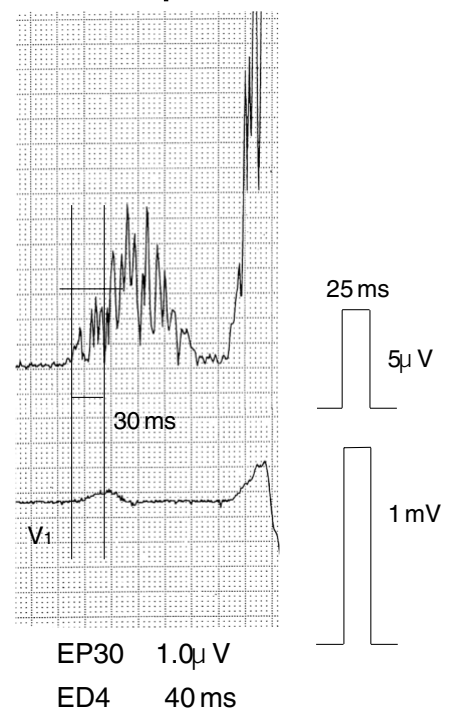

Fig 2. P-SAECG from Group C (Left) and Group S (Right). The upper column shows a P-SAECG and the lower column shows the body surface ECG with leads II and $\mathrm{V}_{1}$. The solid vertical lines indicate the starting point of the filtered $\mathrm{P}$ wave and the point $30 \mathrm{~ms}$ from the onset. The solid horizontal line represents $4 \mu \mathrm{V}$. Group $\mathrm{S}$ has a lower EP30 and longer ED4 than Group C (see text for abbreviations).

point of a filtered $\mathrm{P}$ wave was defined as the point at which the amplitude first exceeded $1 \mu \mathrm{V}$.

EPS After obtaining written informed consent from all the patients, an EPS was performed under anesthesia induced by intravenous propofol infusion. Two $6 \mathrm{~F}$ catheters with 4 electrodes were inserted though the left femoral vein: one was placed at the high right atrium and the other was placed at the right ventricular apex. A $6 \mathrm{~F}$ catheter with 8 electrodes was inserted through the left femoral vein, and placed for His bundle-electrogram recording. To record the $\mathrm{SNE}$, an $8 \mathrm{~F}$ sheath $(85 \mathrm{~cm}$ length) was inserted through the right femoral vein into the right atrium. A $5 \mathrm{~F}$ catheter with 10 electrodes was then inserted into the right atrium through this sheath. This catheter had recording 9 electrodes at the

Table 1 Parameters of P-wave Signal-Averaged Electrocardiogram and Electrophysiological Study

\begin{tabular}{cccc}
\hline \hline & Group C & Group $S$ & p value \\
\hline P-SAECG & & & \\
EP30 $\mu \mathrm{V})$ & $3.2 \pm 1.7$ & $2.1 \pm 1.0$ & $<0.05$ \\
ED4 $(\mathrm{ms})$ & $19.7 \pm 12.0$ & $35.6 \pm 16.4$ & $<0.05$ \\
EPS & & & \\
$P-A H(\mathrm{~ms})$ & $50.3 \pm 8.2$ & $48.0 \pm 7.0$ & $N S$ \\
AS-P $(\mathrm{ms})$ & $4.8 \pm 3.3$ & $10.2 \pm 5.5$ & $<0.01$ \\
SACTd $(\mathrm{ms})$ & $77.8 \pm 9.1$ & $103.7 \pm 29.7$ & $<0.05$ \\
SACTi $(\mathrm{ms})$ & $85.8 \pm 17.3$ & $135.2 \pm 35.0$ & $<0.01$ \\
SRT $(\mathrm{ms})$ & $1,250 \pm 456$ & $2,833 \pm 2,102$ & $<0.01$ \\
\hline
\end{tabular}

P-SAECG, $P$ wave signal-averaged electrocardiogram; EP30, root mean square voltages for the initial $30 \mathrm{~ms}$ of the filtered $P$ wave; ED4, duration of low amplitude signals $<4 \mathrm{mV}$ in the initial portion of filtered $P$ wave; EPS, electrophysiological study; $P-A H$, interval from $P$ wave onset to atrial activation on the His bundle-electrogram; AS-P, interval from onset of atrial activation on the sinus-node electrogram to P wave onset; SACTd, direct sino-atrial conduction time; $S A C T i$, indirect sino-atrial conduction time.

tip $(1.0 \mathrm{~mm}$ electrodes separated by $1.5 \mathrm{~mm}$ inter-electrode spacing); the 10th electrode was attached to a point $45-\mathrm{mm}$ proximal to the tip, as an indifferent electrode. The SNE recording catheter was turned over within the superior vena cava, to keep the indifferent electrode indwelling within the superior vena cava, and the recording electrodes were placed on the junction of the superior vena cava and right atrium. The SNE was recorded with the low-end filter set at $0.05-0.1 \mathrm{~Hz}$ and the high-end filter at $40-50 \mathrm{~Hz}$; the degree of amplification was set at $100-200 \mu \mathrm{V} / \mathrm{cm}$. Atrial stimulation was performed via a programmable stimulator (SEC3120 Nihon Kohden Corp, Japan) at twice the diastolic threshold with a pulse-width of $2.0 \mathrm{~ms}$.

Two parameters were measured as indicators of the atrial conduction time: (1) the interval between the onset of atrial activation on the SNE and the P wave onset on the body surface 12-lead ECG during sinus rhythm (AS-P); and (2) the interval between $\mathrm{P}$ wave onset on the 12-lead ECG and the atrial potential on the His bundle-electrogram (P-AH).

The direct sino-atrial conduction time (SACTd) was also measured from the SNE. This was defined as the interval between the onset of the upstroke slope pacemaker pre- 

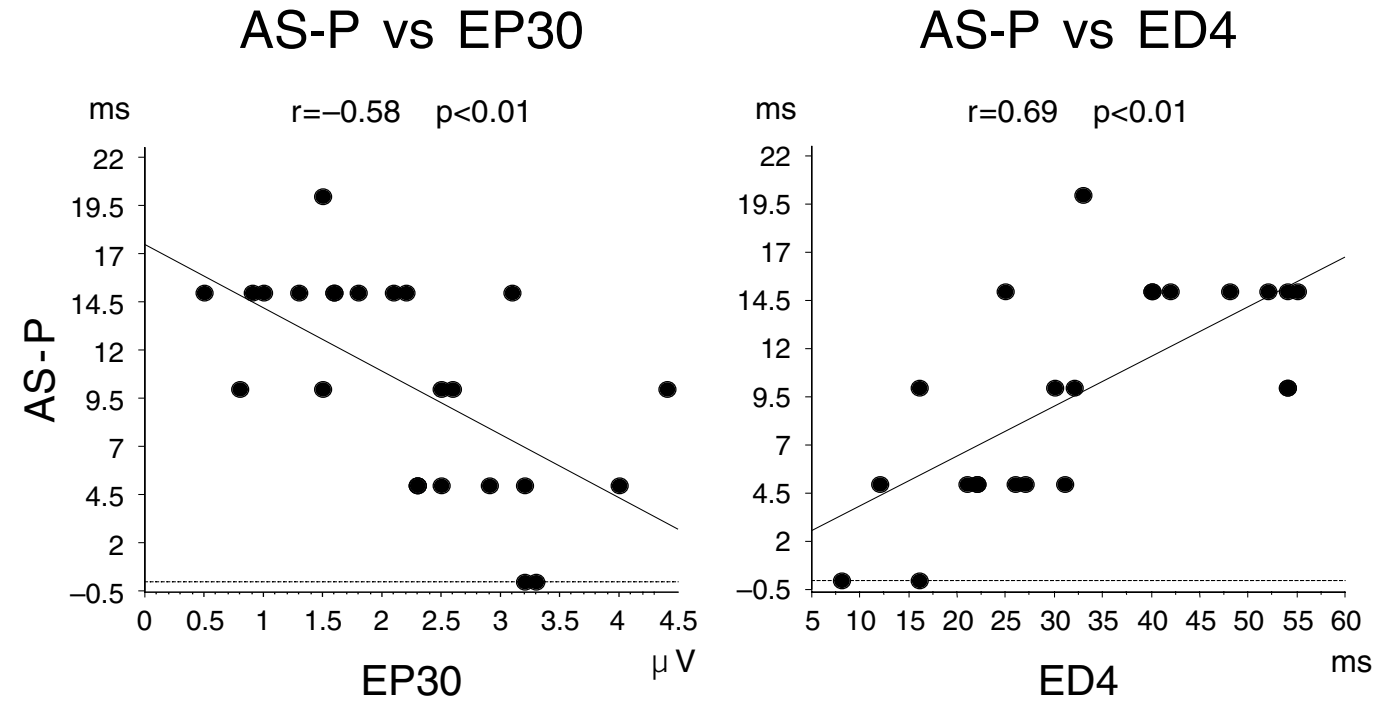

Fig 3. Correlation of AS-P with EP30 and ED4 in Group S. The AS-P is significantly negatively correlated with the $\mathrm{EP} 30(\mathrm{r}=-0.58, \mathrm{p}<0.01)$ and significantly positively correlated with the ED4 $(\mathrm{r}=0.69, \mathrm{p}<0.01)$ (see text for abbreviations).
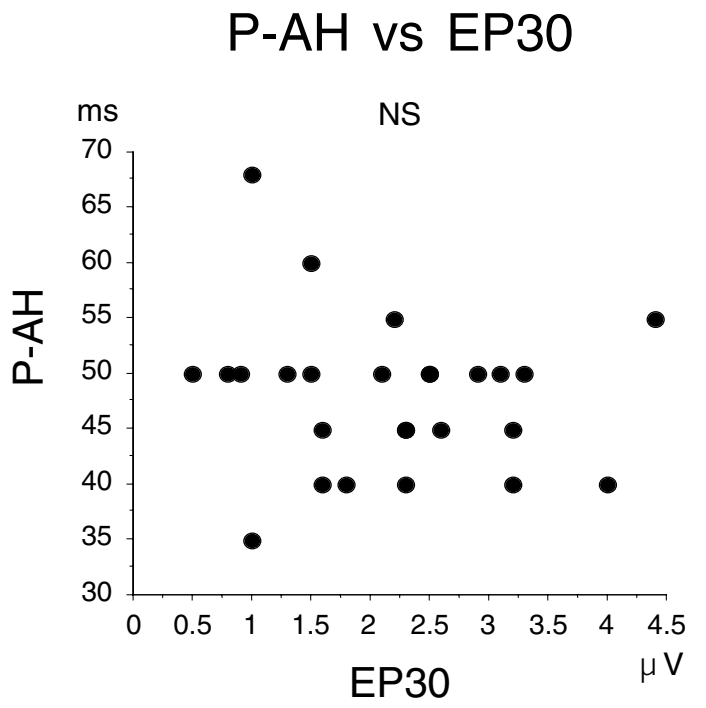

P-AH vs ED4

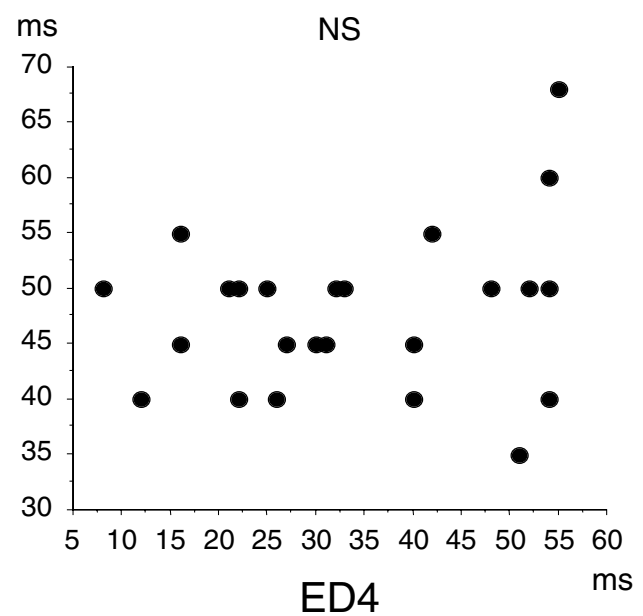

Fig 4. Correlation of P-AH with EP30 and ED4 in Group S. The P-AH is not significantly correlated with the EP30 or the ED4 (see text for abbreviations).

potential on the local electrogram and the onset of rapid atrial depolarization (Fig 1).

The indirect sino-atrial conduction time (SACTi) was measured using the method of Narula et al? The high right atrium was paced for 8 beats at a rate slightly faster than the control sinus rhythm. The SACTi was obtained as half of the interval calculated by subtracting the mean sinus cycle from the interval between the last paced atrial electrogram and the atrial electrogram of the first sinus beat.

These parameters were compared between the 2 groups. Correlations between early components of the filtered $\mathrm{P}$ wave on P-SAECG and EPS parameters were analyzed in Group S.

\section{Statistical Analysis}

All data are expressed as mean $\pm \mathrm{SD}$. The paired t-test and Pearson's coefficient of correlation were used for statistical analysis. Statistical significance was taken as $\mathrm{p}<0.05$.

\section{Results}

\section{$P$-SAECG and EPS Parameters}

Representative P-SAECG findings are shown in Fig 2 and Table 1 shows each of the P-SAECG and EPS parameters. Of the P-SAECG parameters, the EP30 was significantly lower in Group $S$ than in Group C $(2.1 \pm 1.0$ vs $3.2 \pm$ $1.7 \mu \mathrm{V}, \mathrm{p}<0.05)$, and the ED4 was significantly longer in Group S than in Group C $(35.6 \pm 16.4$ vs $19.7 \pm 12.0 \mathrm{~ms}, \mathrm{p}<$ $0.05)$. Of the EPS parameters, the AS-P was significantly longer in Group $S$ than in Group C $(10.2 \pm 5.5$ vs $4.8 \pm 3.3 \mathrm{~ms}$, $\mathrm{p}<0.01)$, and the P-AH did not differ significantly between both groups ( $50.3 \pm 8.2$ vs $48.0 \pm 7.0 \mathrm{~ms})$. The SACTi was significantly longer in Group $S$ than in Group C $(135.2 \pm 35.0$ vs $85.8 \pm 17.3 \mathrm{~ms}, \mathrm{p}<0.01)$ and SACTd was also significantly longer in Group $S$ than in Group C $(103.7 \pm 29.7$ vs $77.8 \pm$ $9.1 \mathrm{~ms}, \mathrm{p}<0.01)$. 

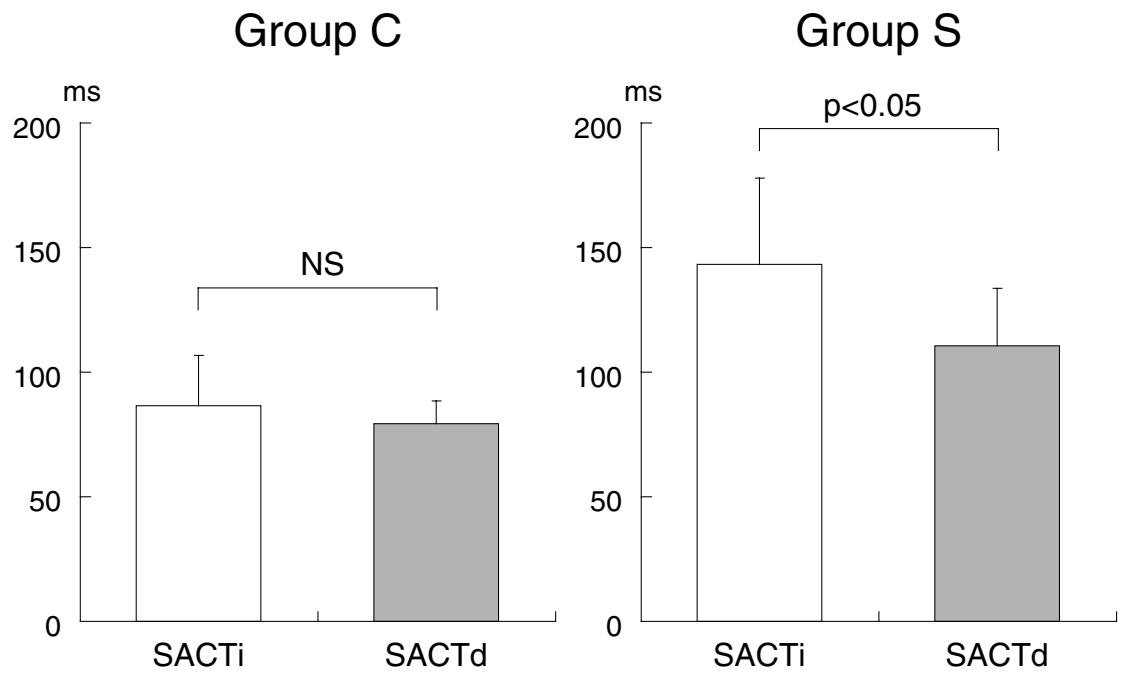

Fig 5. Comparison between the SACTi and SACTd. (Left) Group C, (Right) Group S. In Group $C$ the SACTi does not differ significantly from the SACTd; in Group S it is longer (see text for abbreviations).

SACTi vs EP30

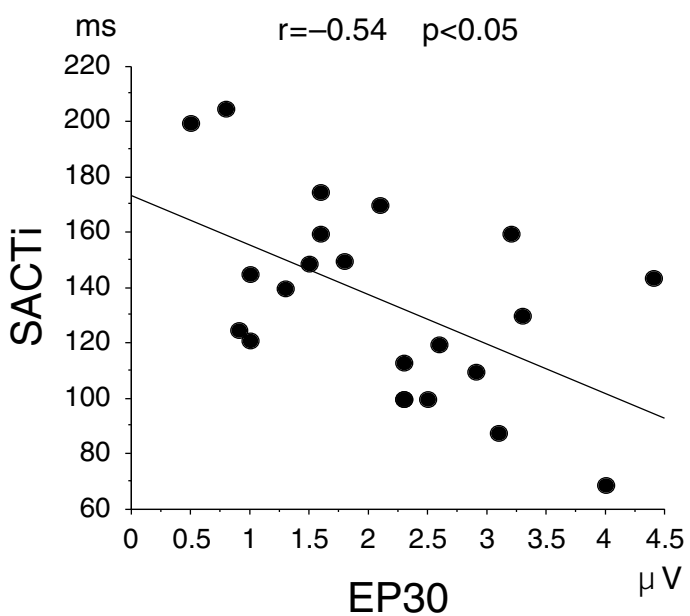

SACTi vs ED4

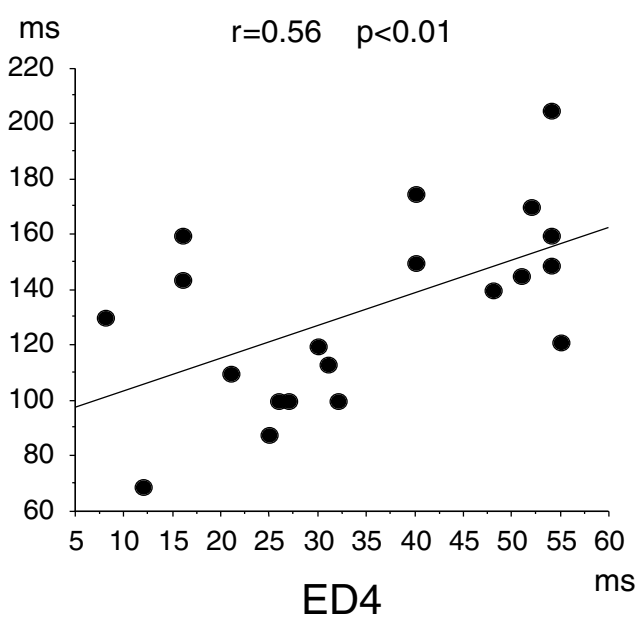

Fig 6. Correlation of the SACTi with EP30 and ED4 in Group S. The SACTi is significantly negatively correlated with the EP30 $(r=-0.54, p<0.05)$ and significantly positively correlated with the ED4 ( $r=0.56, p<0.01)$ (see text for abbreviations).

SACTd vs EP30

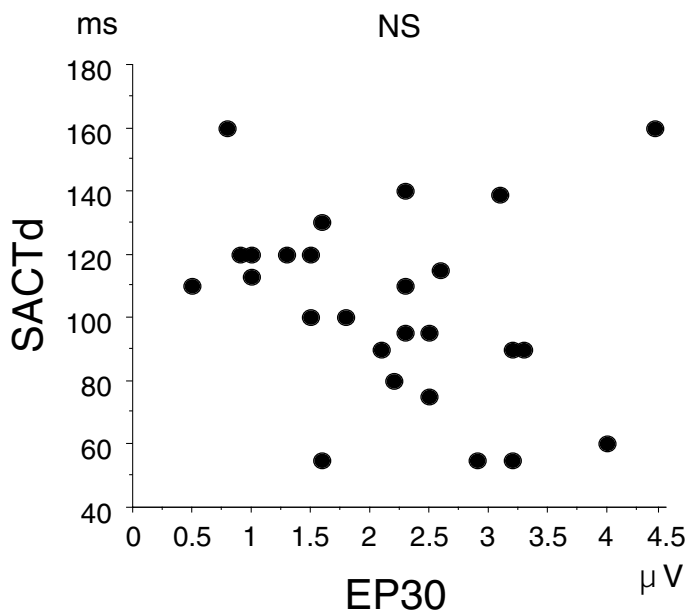

SACTd vs ED4

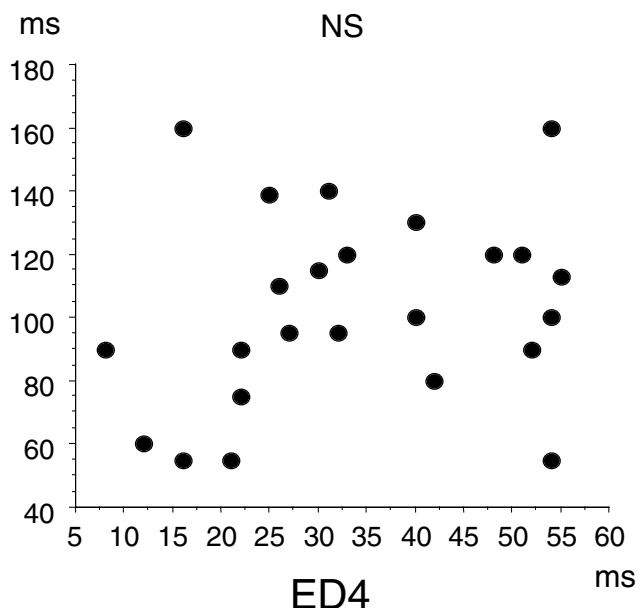

Fig 7. Correlation of the SACTd with EP30 and ED4 in Group S. The SACTd is not significantly correlated with the EP30 or the ED4 (see text for abbreviations). 
Relationship Between Atrial Conduction Time (AS-P, P-AH) and Early Components of the Filtered P Wave on P-SAECG (EP30, ED4) in Group $S$

Fig 3 shows the relationship between the AS-P and EP30 and ED4 in Group S. There is a significant negative correlation between the AS-P and EP30 $(r=-0.58, \mathrm{p}<0.01)$ and a significant positive correlation between the AS-P and ED4 $(\mathrm{r}=0.69, \mathrm{p}<0.01)$. In contrast, the P-AH was not significantly correlated with the EP30 or ED4 in Group S (Fig 4).

\section{Comparison of the SACTi and SACTd}

Fig 5 shows the results of a comparison of SACTi with SACTd. In Group C, there was no significant difference between the SACTi and SACTd, but in Group S the SACTi was significantly longer than the SACTd $(\mathrm{p}<0.05)$.

\section{Relationship of SACTi and SACTd to Early Components of the Filtered P Wave (EP30, ED4) in Group $S$}

Fig 6 shows the relation between the SACTi and EP30 and ED4 in Group S. There was a significant negative correlation between the SACTi and EP30 $(r=-0.54, p<0.05)$ and a significant positive correlation between the SACTi and ED4 $(r=0.56, p<0.01)$. Fig 7 shows the relation between the SACTd and EP30 and ED4 in Group S. In contrast, the SACTd was not significantly correlated with the EP30 or ED4.

\section{Discussion}

It is not difficult to diagnose SSS in the event of transient cardiac arrest or sinus bradycardia documented on an ECG recording, which corresponds to symptoms of brain ischemia such as dizziness or syncope, and to symptoms of heart failure such as shortness of breath or easily induced fatigue. When no episodes can be detected by noninvasive evaluation using 24-h ambulatory monitor ECG or ECG monitoring, however, it is sometimes necessary to make an invasive electrophysiological evaluation of sino-atrial node function, using the over drive suppression test ${ }^{10,11}$ and the ATP load test $!^{2,13}$

Yamada et al recently reported that the initial portion of the filtered P wave on a P-SAECG had significantly lower amplitude in patients with SSS than in patients with normal sino-atrial node function, which is a useful difference indicating SSS5 They found that the sensitivity of this method for the diagnosis of SSS was $76 \%$ and the specificity $91 \%$, if an abnormally low amplitude is defined by an EP30 $<3.0 \mu \mathrm{V}$ and ED4 $>22 \mathrm{~ms}$. Yamada et al further prospectively studied the relationship between the characteristics of the initial portion of the filtered $\mathrm{P}$ wave and sino-atrial node dysfunction in patients with paroxysmal AF. They found that the initial portion of the filtered $\mathrm{P}$ wave was useful for predicting sino-atrial node dysfunction ${ }^{14}$ In the present study, the SSS group had a significantly lower EP30 and significantly longer ED4 than the normal sinoatrial node function group, endorsing the findings of Yamada et al.

In previous EPS using the SNE, we found that the major lesion responsible for SSS was located in the transitional zone close to the sino-atrial node. Transient episodes of cardiac arrest in patients with SSS are attributable to sinoatrial block caused by disturbed conduction around the node!5,16 Katoh et al reported that the monophasic action potential duration at the crista terminalis in the sinus node disease group were significantly longer than in the control group. These findings suggest an electrophysiological abnormality at the crista terminalis in SSS ${ }^{17}$ Histopathologic studies show that patients with SSS have a decreased number of sino-atrial node cells, as well as steatosis, fibrosis and other changes in the tissues surrounding the sino-atrial node ${ }^{18-20}$ Accordingly, we believe that patients with SSS have electrophysiological abnormalities that delay sinoatrial conduction and involve atrial tissue around the sinoatrial node.

We hypothesized that the disturbed conduction in the atrial myocardium around the sino-atrial node was reflected as the initial low potential of the P wave of the P-SAECG rather than the SACT. We analyzed the atrial conduction time by measuring 2 parameters. The first parameter is the AS-P, which measures the interval from atrial activation on the SNE (the earliest atrial activation site) to the onset of the $\mathrm{P}$ wave. The second parameter is the $\mathrm{P}-\mathrm{AH}$, which measures the interval from the $\mathrm{P}$ wave on the body surface ECG to the atrial potential on His bundle electrograms. The AS-P appears to reflect the initial conduction time of the atrial tissue around the sino-atrial node, and the $\mathrm{P}-\mathrm{AH}$ reflects the subsequent conduction time of the entire right atrium. When the AS-P and P-AH for Group C and Group $\mathrm{S}$ were compared, the AS-P was significantly longer in Group $\mathrm{S}$ and the P-AH did not differ significantly between the groups. These electrophysiological findings indicate that, in patients with SSS, atrial conduction is delayed in a relatively limited area of atrial tissue around the sino-atrial node.

We then analyzed the relationship between the AS-P and $\mathrm{P}-\mathrm{AH}$, as electrophysiological parameters of atrial conduction, and EP30 and ED4, as the P-SAECG parameters, in Group S. AS-P was negatively correlated with the EP30 and positively with ED4, whereas the P-AH showed no significant correlation with either EP30 or ED4. These results suggest that patients with SSS have disturbed conduction in the atrial myocardium around the sino-atrial node. The resulting decrease in excited atrial myocardium during the early phase of the $\mathrm{P}$ wave is responsible for the abnormal amplitude signals in the early phase on the P-SAECG. The $\mathrm{P}-\mathrm{AH}$ appears to reflect the conduction time of the right atrium after activation of the atrial myocardium around the sino-atrial node, which probably explains why the $\mathrm{P}-\mathrm{AH}$ is uncorrelated with the parameters of the early-phase PSAECG.

We measured the sino-atrial conduction time by 2 methods. The SACTi was measured by the method of Narula, and the SACTd was measured via the SNE recordings. In Group $C$ there was no significant difference between the SACTi and SACTd, whereas in Group S the SACTi was significantly longer than the SACTd. In Narula's method, the sino-atrial conduction time is calculated from the difference between the first cycle length after stimulation and the sinus cycle length before stimulation, recorded by the pacing electrode of the atrial muscle near the sino-atrial node? Conduction through the atrial myocardium near the sino-atrial node is disturbed in patients with SSS. Delayed conduction between the stimulated site and the sino-atrial node can therefore lead to overestimation of the sino-atrial conduction time by the pacing method. This probably explains why the SACTi was significantly longer than the SACTd in patients with SSS, whereas no difference was observed in the normal controls.

Yamada et al reported that the SACTi calculated using Narula's method was negatively correlated with the EP30 
and positively correlated with the ED4, and that abnormalities of these early components of the $\mathrm{P}$ wave on the PSAECG reflect disturbed sino-atrial conduction? We also found that the SACTi was significantly correlated with the EP30 and ED4, consistent with the findings of Yamada et al. However, we found no significant correlation between the SACTd and either the EP30 or ED4. Delayed conduction through the atrial myocardium in patients with SSS becomes more pronounced with increasing damage of the atrial muscle near the sino-atrial node, resulting in a longer SACTi. The amplitude of the initial portion of the filtered $P$ wave on the P-SAECG was thought to decrease with increasing damage to the atrial myocardium around the sino-atrial node. This probably explains why the initial portion of the filtered $\mathrm{P}$ wave was correlated with the SACTi but not with the SACTd, which is unaffected by the atrial conductivity and indicate the true sino-atrial conduction time.

We have demonstrated that the initial portion of the $\mathrm{P}$ wave on the P-SAECG reflects the conductivity of the atrial tissue around the sino-atrial node. And we conclude that the P-SAECG would be useful noninvasive examination for identification of patients with severe transient cardiac arrest who have disturbed conduction around the sinoatrial node. However, it remains to be investigated, using a large number of subjects, whether the early initial portion of the P wave on the P-SAECG reflects the degree of disturbed stimulus conduction through the atrial myocardium around the sino-atrial node, so that the P-SAECG could be used to predict the severity of the SSS.

\section{Conclusion}

The reduced amplitudes of the initial portion of the $\mathrm{P}$ wave on the P-SAECG, which are observed in patients with SSS, have been shown to be independent of the efficiency of conduction within the sino-atrial node, and are due to disturbed conduction of the atrial myocardium around the node. The characteristics of the initial portion of the $P$ wave on the P-SAECG are therefore a useful noninvasive indicator of SSS.

\section{References}

1. Fukunami M, Yamada T, Ohmori M, Kumagai K, Umemoto K, Sakai A, et al. Detection of patients at risk for paroxysmal atrial fibrillation during sinus rhythm by $\mathrm{P}$ wave-triggered signal-averaged electrocardiogram. Circulation 1991; 83: 162-169.

2. Murakami Y, Okamoto M, Nakayama R. Atrial late potential by P wave-triggered signal-averaged electrocardiogram and paroxysmal atrial fibrillation. Nippon Rinsho 1995; 53: 489-495.
3. Engel TR, Vallone N, Windle J. Signal-averaged electrocardiograms in patients with atrial fibrillation or flutter. Am Heart J 1988; 115: $592-597$.

4. Okumura Y, Watanabe I, Nakai T, Sugimura H, Hashimoto K, Masaki R, et al. Recurrence of atrial fibrillation after internal cardioversion of persistent atrial fibrillation. Circ J 2005; 69: 1514-1520.

5. Yamada T, Fukunami M, Kumagai K, Abe Y, Kim J, Sanada S, et al. Detection of patients with sick sinus syndrome by use of low amplitude potentials early in filtered P wave. J Am Coll Cardiol 1996; 28: $738-744$.

6. Reiffel JA, Gang E, Gliklich J, Weiss MB, Davis JC, Patton JN, et al. The human sinus node electrogram: A transvenous catheter technique and a comparison of directly measured and indirectly estimated sinoatrial conduction time in adults. Circulation 1980; 62: 1324-1334.

7. Gomes JAC, Kang PS, El-Sherif N. The sinus node electrogram in patients with and without sick sinus syndrome: Techniques and correlation between directly measured and indirectly estimated sinoatrial conduction time. Circulation 1982; 66: 864-873.

8. Rubenstein JJ, Schulman CL, Yurchak PM, DeSanctis RW. Clinical spectrum of the sick sinus syndrome. Circulation 1972; 46: 5-13.

9. Narula OS, Shantha N, Vasquez M, Towne WD, Linhart JW. A new method for measurement of sinoatrial conduction time. Circulation 1978; 58: 706-714.

10. Mandel W, Hayakawa H, Danzig R, Marcus HS. Evaluation of sinoatrial node function in man by overdrive suppression. Circulation 1971; 44: 59-66.

11. Kasanuki H. Electrophysiological and clinical study of sick sinus syndrome using the overdrive suppression test. Jpn Circ J 1980; 44: $505-517$.

12. Imai S, Yagi H, Uenishi T, Kanmatsuse K. Effect of ATP on sinoatrial and atrioventricular conduction, evaluated by direct sinus node electrograms. J Nihon Univ Med 1996; 38: 9-19.

13. Aoyama H, Imai S, Yagi H, Kanmatsuse K. The effect of ATP on sinus nodal function, and the usefulness for diagnosis of sick sinus syndrome - evaluated by sinus node electrograms-. Jpn J Electrocardiology 2001; 22: 16-24.

14. Yamada T, Fukunami M, Shimonagata T, Kumagai K, Asano Y, Hirata A, et al. Identification of sinus node dysfunction by use of $\mathrm{P}$-wave signal-averaged electrocardiograms in paroxysmal atrial fibrillation: A prospective study. Am Heart J 2001; 142: 286-293.

15. Yagi H, Suzuki H, Sugino K, Watanabe T, Takahashi N, Uenishi T, et al. Clinical significance of overdrive suppression on sinus node automaticity and sino-atrial conduction, and influence of autonomic nervous system on overdrive suppression: Evaluation by sinus node electrograms. Jpn J Electrocardiol 1996; 16: 360-368.

16. Yagi H, Uenishi T, Sugino K, Suzuki H, Kanmatsuse K. Sinus node automaticity and sinoatrial conduction evaluated by direct sinus node electrograms. Shinzou 1997; 29: 251-259.

17. Katoh H, Shinozaki T, Baba S, Satoh S, Kagaya Y, Watanabe J, et al. Monophasic action potential duration at the crista terminalis in patients with sinus node disease. Circ J 2005; 69: 1361 - 1367.

18. Thery C, Gosselin B, Lekieffre J, Warembourg H. Pathology of sinoatrial node: Correlations with electrocardiographic findings in 111 patients. Am Heart J 1977; 93: 735-740.

19. Sugiura M, Ohkawa S. A Clinicopathologic study on sick sinus syndrome with histological approach to the sinoatrial node. Jpn Circ J 1980; 44: 497-504.

20. Ohkawa S. Clinicopathologic correlations in various kinds of arrhythmias with special references to the histopathology of the conduction system. J Arrhythmia 2000; 16: 312-329. 